Clipper F. Young*, PharmD, MPH, CDCES, BC-ADM, BCGP, Rachael Mullin, OMS IV, Joy A. Moverley, MPH, PA-C, DHSC and Jay H. Shubrook, DO

\title{
Associations between diabetes-related distress and predicted cardiovascular complication risks in patients with type 2 diabetes
}

https://doi.org/10.1515/jom-2021-0204

Received August 13, 2021; accepted January 14, 2022;

published online February 22, 2022

\section{Abstract}

Context: Diabetes-related distress (DRD) is experienced by nearly $50 \%$ of people with diabetes at any given time in their diagnosis. The effects of low socioeconomic status (SES) and lacking access to resources can increase DRD. In addition, cardiovascular (CV) complications associated with diabetes are associated with higher DRD scores.

Objectives: This study evaluated the associations between DRD and predicted CV risks in participants with type 2 diabetes.

Methods: This cross-sectional study included 234 individuals with low SES who were Medi-Cal (California version of Medicaid) beneficiaries and sought medical care at a safety-net clinic system. The Problem Areas in Diabetes (PAID) questionnaire assessed DRD levels. The United Kingdom Prospective Diabetes Study Risk Engine was utilized to predict 10-year risks for coronary heart disease (CHD), fatal CHD, stroke, and fatal stroke. A multivariate linear regression model was constructed between the two variables, including other variables to control for potential confounding factors, for assessing the associations.

Results: After controlling for potential confounders, participants' total PAID questionnaire scores were significantly

${ }^{*}$ Corresponding author: Clipper F. Young, PharmD, MPH, CDCES, BCADM, BCGP, Primary Care Department, College of Osteopathic Medicine, Touro University California, 1310 Club Drive, Vallejo, CA 94592, USA, E-mail: cyoung6@touro.edu. https://orcid.org/00000002-5313-7866

Rachael Mullin, OMS IV, College of Osteopathic Medicine, Touro University California, Vallejo, CA, USA

Joy A. Moverley, MPH, PA-C, DHSc, College of Education and Health Sciences, Joint MSPAS/MPH Program, Touro University California, Vallejo, CA, USA

Jay H. Shubrook, DO, Primary Care Department, College of Osteopathic Medicine, Touro University California, Vallejo, CA, USA associated with their 10-year predicted fatal CHD risks ( $\mathrm{B}=0.060,95 \% \mathrm{CI}$ : [0.00084, 0.12], $\mathrm{p}=0.047)$.

Conclusions: After controlling for covariates, DRD levels exhibited a significant association with increased 10-year predicted fatal CHD risks in patients with type 2 diabetes and lower SES. Screening for DRD and provision of appropriate psychosocial interventions may reduce the risks of CHD in those with type 2 diabetes.

Keywords: cardiovascular complications; diabetes-related distress; psychosocial aspect of diabetes management; type 2 diabetes.

The psychological impact of managing diabetes has been well established in the medical literature, with multiple studies illustrating a negative relationship between diabetes-related distress (DRD) and diabetes self-management decisions [1, 2]. The term "DRD" is to describe patients' psychological and behavioral responses to the diagnosis, management, and fear of complications associated with diabetes [3]. In 2016, the American Diabetes Association (ADA) released a position statement endorsing the use of validated screening tools to assess DRD by incorporating psychological interventions to address perceived gaps in psychological care and encourage optimal medical outcomes [4]. These gaps are often even larger in populations of lower socioeconomic status (SES) because of the strong link between mental and physical health [5]. Experiencing a lower SES often means access to fewer resources, including healthcare and mentalhealth-related ones. The lack of access often intensifies the distress experienced by those with DRD. This distress is only compounded by the management of a complicated chronic condition such as type 2 diabetes [5].

The Diabetes Attitudes Wishes and Needs (DAWN) study conducted in 2005 brought the prevalence and the impact of DRD to light. The study demonstrated that $41 \%$ of patients with diabetes $(n=5,104)$ reported low perceived well-being [6]. Many providers recognized that patients' concerns relating to the condition negatively impacted the outcomes of 
diabetes management [6]. As one analysis of nearly 500,000 participants found with an average follow-up of 5.8 years, people with diabetes have a $25 \%$ increased risk of depression compared to participants without diabetes, which makes the importance of identifying, addressing, and managing distress among patients with diabetes particularly important [7]. This same trend has been associated between cardiovascular disease (CVD) and depression as well. Similarly, the second DAWN study (DAWN2), published in 2013, highlighted the importance of identifying DRD, revealing that study participants $(n=8,596)$ believed their diabetes had negatively impacted their physical health (62.2\%) and emotional well-being (46.2\%) [8]. For a patient with diabetes, the necessary lifestyle modifications and long-term commitment to treatment are likely to be mentally taxing. This may create an environment in which it is more difficult for patients to manage their diabetes, leading to poorer physical health. Due to the complexity of this issue, early intervention is key to ensure minimizing the impact resulting from what this negative feedback loop can cause.

Previous studies have demonstrated the impact of DRD on long-term health outcomes [1, 2]. Fisher and colleagues $[1,2]$ investigated the cross-sectional and longitudinal relationships between DRD and HbA1c. These researchers determined that DRD exhibited significant time concordant relationships with $\mathrm{HbA1c}$, suggesting that emotional distress secondary to diabetes management directly impacted diabetes clinical outcomes [1, 2]. Another study concluded that CVD risk factors were significantly associated with DRD [9]. In addition, DRD was the factor found to associate significantly with suboptimal glycemic management after adjusting for covariates [9].

A meta-analysis and systematic review totaling 346,037 people with diabetes and depression, and 614,574 with diabetes only, demonstrated that emotionally distressed patients with type 2 diabetes exhibit an increase in cardiovascular (CV) morbidity and mortality rates [10]. This meta-analysis found statistically significant findings, including a $48 \%$ increase in CV mortality, a $37 \%$ increase in CHD, and a 33\% increase in stroke risk [10]. Comparably, there is less evidence in the literature on DRD as a separate risk factor for $\mathrm{CV}$ complication risks. A pilot study at a safety-net clinic in Northern California $(\mathrm{n}=48)$ revealed significant associations between DRD and predicted 10-year fatal and nonfatal CHD in patients with type 2 diabetes [11]. Several hypotheses have been proposed to explain this phenomenon, such as endothelial microparticles triggering pro-inflammatory signaling cascades, leading to endothelial damage and subsequent CVD [12]. Regardless of the underlying mechanism that causes CVD, the identification of DRD is an essential step in managing patients' psychological health and preventing the future development of CVD [12]. This study evaluated the associations between DRD and predicted CV risks in participants with type 2 diabetes. Thus, the authors hypothesized that significant associations exist between DRD and predicted 10-year CV risks in patients with type 2 diabetes.

\section{Methods}

\section{Study design}

This study employed an observational, cross-sectional design for data collection through a survey instrument, enrolling those seeking medical attention at two federally qualified health clinics, both Solano County Family Health Services, in Vallejo and Fairfield, CA. The Institutional Review Board at Touro University California and Solano County Family Health Services approved the research protocol (IRB \# P-2314). All participants provided written informed consent on paper by the research team, including principal investigators and student researchers. The study received intramural funding by Touro University California, and each study participant was compensated for their time in the form of a gift card.

\section{Setting}

A member of the research team interviewed each participant in a patient room either before or after their clinic encounter to collect information utilizing the survey instrument. Data collection was performed from May 2015 to September 2017.

\section{Participants}

The inclusion criteria included patients: (1) with type 2 diabetes; (2) between 40 and 85 years of age; (3) who had at least one visit at a study site in the past year; and (4) who had been prescribed and taking at least one antidiabetic medication. The exclusion criteria included: (1) history of stroke and/or heart attack; (2) not able to complete or understand the Problem Areas in Diabetes (PAID) questionnaire (excluded after the interview); (3) insufficient information to calculate the complication risks at the point when the participant's electronic medical record was reviewed; and (4) a self-reported total PAID score of 0 (indicating no DRD from having to manage diabetes, thus excluded after the interview). No study participant was excluded due to having a self-reported total PAID score of 0 . Potential subjects who presented to this safety-net clinic system for diabetes consultation services or primary care services were asked to participate in the study following the inclusion criteria. This clinic system only accepts patients with Medi-Cal health insurance coverage, and most Medi-Cal beneficiaries must meet the Medicaid income/financial eligibility at or below a certain threshold for receiving the health insurance coverage. Thus, most patients who seek medical care at this clinic system have lower SES, defined by and measurable via income, education level, and occupation.

This study's primary outcomes were to identify associations between the total PAID scores (an indicator of DRD level; independent 
variable) and the four levels of 10-year CV complication risks (dependent variable). Potential confounders were identified as depression status, systolic blood pressure, BMI, tobacco use, LDL-C levels, gender, and age.

The initial gathering of demographic information was from the clinic system's electronic medical records (EMRs), and the gathered information was confirmed with the participant before the interview for administering the study survey. Information relating to race/ethnicity was obtained within the "Patient Information" section in the EMR.

\section{Data sources}

This study utilized the PAID questionnaire to quantify participants' selfreported levels of DRD. The PAID questionnaire includes 20 questions about emotional distress, self-care behavior, and perceived diabetes burden [13]. The research team also utilized the United Kingdom Prospective Diabetes Study (UKPDS) Risk Engine 2.0 to predict 10-year fatal and nonfatal stroke and CHD risks for participants with diabetes [14].

The survey instrument's demographic data included age, sex, SES, ethnicity, exercise patterns, and current medical history. The SES was determined by proxies, such as education level, occupation, and income. For this study, the research team specifically utilized the occupation component for categorizing SES; the Nam-Powers-Boyd Occupational Status Scale was utilized to assess participants' occupations [15]. The reason for choosing occupation as the primary proxy was because occupation, to a certain extent, is a reflection of education, income, and social status as well as a demonstration of relative rank in social hierarchies. For the same reason, a participant's occupation has been utilized as a proxy to predict health outcomes in health studies [16].

The occupation status scale scores were stratified into three tiers: low (1-33), medium (34-66), and high (67-100). Individual annual income was categorized as: $<\$ 20,000 ; \$ 20,000$ to $\$ 34,999 ; \$ 35,000$ to $\$ 49,999 ; \$ 50,000$ to $\$ 75,000$; or $>\$ 75,000$. Ethnicity categorization was based on the UKPDS Risk Engine 2.0 with the categories: White, Afro-Caribbean, and Asian-Indian [14].

\section{Study sample size}

The study sample size was predetermined to be 206 with an alpha level at 0.05 , power at 0.95 , and squared partial correlation at 0.09 , and both numbers of the test and control covariates were set at 5 .

\section{Quantitative variables}

The predicated 10-year CV risks from the UKPDS Risk Engine 2.0 were reported in continuous data. This risk engine included two components: inputs and outputs. Input items included age, duration of diabetes, sex, atrial fibrillation status, ethnicity, smoking status, HbA1c, systolic blood pressure, total cholesterol, and HDL cholesterol; the four outputs were CHD, fatal CHD, stroke, and fatal stroke [14].

The information captured by the PAID questionnaire was in categorical data. The 20 questions in this instrument were in Likert-scale format with five options/scoring categories, e.g., "not a problem" (0), "minor problem" (1), "moderate problem" (2), "somewhat serious problem (3), and "serious problem" (4) [13]. A linear transformation process was performed, converting the data to continuous (e.g., summing the scores from all the questions and multiplying by 1.25 with a maximum score of 100). The exact statements on the PAID questionnaire can be found in the publication in 1995 by Polonsky and colleagues [13].

\section{Statistical methods}

To link DRD levels to predicted 10-year CV complication risks, the research team analyzed the data obtained from both instruments and constructed statistical models to examine the relationships between PAID scores and predicted 10-year CV risks. Statistical analyses were performed utilizing STATA Version 14.1 (College Station, TX). One simple linear regression model and one multivariate linear regression model were constructed between the two variables. The covariates included in the multivariate linear regression model were depression status, ethnicity, gender, age, insulin usage, number of diabetes medications, annual income, occupational index score, systolic blood pressure, BMI, and tobacco use.

\section{Results}

\section{Demographics: participants and descriptive data}

A total of 280 participants were interviewed, and survey data were collected from these individuals. Forty-six participants were subsequently excluded due to missing clinical data from their electronic medical records or exhibiting a zero on the PAID questionnaire (indicating no DRD). Among the 234 participants included in this study's analyses, $47.9 \%$ were male and $52.1 \%$ female. The study setting was a racially diverse setting, with Afro-Caribbean being the most common ethnicity $(38.9 \%)$ in the sampled population. [Note: The UKPDS Risk Engine provided three categories of "ethnicity": White, AfroCaribbean, and Asian-Indian.] The mean age was 56.7 years (ranging from 40 to 81 ), $54.3 \%$ of the participants either have smoked in the past or currently smoke, and $27.8 \%$ expressed a known depression status. A majority of the participants expressed participating in some form of physical activity at least 3 days a week (62.8\%), utilizing insulin (60.7\%), and having systolic blood pressure $<140 \mathrm{mmHg}(65.0 \%)$. These baseline characteristics are summarized in Table 1.

\section{Social characteristics}

According to the Nam-Powers-Boyd Occupational Status Scale, the average occupational index score was 35.5 (ranging from 1 to 94), which is equivalent to the occupational score of a receptionist in an administrative office or a bartender [15]. The maximum score on this scale is 100.

\section{Clinical characteristics}

The mean total PAID score was $22 \pm 16.7$ points (ranging from 1.25 to 78.5 ). The average BMI among all the 
Table 1: Demographic characteristics $(n=234)$.

\begin{tabular}{|c|c|c|}
\hline Characteristics & Count & $\begin{array}{l}\text { Percentage, } \\
\%\end{array}$ \\
\hline \multicolumn{3}{|l|}{ Gender } \\
\hline Male & 112 & 47.9 \\
\hline Female & 122 & 52.1 \\
\hline \multicolumn{3}{|l|}{ Ethnicity } \\
\hline White & 80 & 34.2 \\
\hline Afro-Caribbean & 91 & 38.9 \\
\hline Asian-Indian & 63 & 26.9 \\
\hline \multicolumn{3}{|l|}{ Occupational index score } \\
\hline Low (1-33) & 134 & 57.3 \\
\hline Medium (34-66) & 67 & 28.6 \\
\hline High (67-100) & 33 & 14.1 \\
\hline \multicolumn{3}{|l|}{ Individual annual income } \\
\hline$<\$ 20,000$ & 195 & 83.3 \\
\hline$\$ 20,000$ to $\$ 34,999$ & 23 & 9.8 \\
\hline$\$ 35,000$ to $\$ 49,999$ & 12 & 5.1 \\
\hline$\$ 50,000$ to $\$ 75,000$ & 3 & 1.3 \\
\hline$>\$ 75,000$ & 1 & 0.4 \\
\hline $\begin{array}{l}\text { Count, \% } \\
\text { Smoking status }\end{array}$ & Yes & No \\
\hline Current & $55(23.5)$ & $107(45.7)$ \\
\hline Prior & $72(30.8)$ & \\
\hline $\begin{array}{l}\text { Physical activities (at least three days per } \\
\text { week) }\end{array}$ & $\begin{array}{l}147 \\
(62.8)\end{array}$ & $87(37.2)$ \\
\hline Depression status & $65(27.8)$ & $169(72.2)$ \\
\hline Insulin use & $\begin{array}{l}142 \\
(60.7)\end{array}$ & $92(39.3)$ \\
\hline Systolic blood pressure $<140 \mathrm{mmHg}$ & $\begin{array}{l}152 \\
(65.0)\end{array}$ & $82(35.0)$ \\
\hline
\end{tabular}

participants was $33.5 \pm 8.1 \mathrm{~kg} / \mathrm{m}^{2}$ (ranging from 20 to 63 ). The mean HbA1c was $9.0 \pm 2.3 \%$ (ranging from 5.5 to $16.5 \%$ ), and the mean LDL-C level was $97 \pm 45 \mathrm{mg} / \mathrm{dL}$ (ranging from 18 to $400 \mathrm{mg} / \mathrm{dL}$ ). Participants had an average duration of diabetes of $10.0 \pm 7.9$ years (ranging from 0.25 to 35 years). Regarding the 10 -year predicted risks for cardiovascular complications utilizing the UKPDS Risk Engine, participants in this study had an average risk for CHD of $14.6 \%$ and an average fatal CHD risk of $10.4 \%$. With the exclusion of outliers, the mean stroke risk was $6.7 \%$, and the mean fatal stroke risk was $1.0 \%$. The descriptive statistics for these parameters, as well as for other lipid panel parameters, are summarized in Table 2. Table 3 summarizes stratified data based on the baseline characteristics and the UKPDS Risk Engine outputs. In addition, Table 4 shows the correlation coefficients between stratified PAID scores and the UKPDS Risk Engine outputs.
Table 2: Descriptive statistics ( $n=234)$.

\begin{tabular}{lrrrr}
\hline & Mean & SD & Minimum & Maximum \\
\hline Total PAID score & 22.0 & 16.7 & 1.25 & 78.75 \\
Age, years & 56.7 & 8.5 & 40 & 81 \\
Occupational index score & 35.5 & 23.1 & 1 & 94 \\
BMI & 33.5 & 8.1 & 20 & 63 \\
HbA1c & 9.0 & 2.3 & 5.5 & 16.5 \\
Duration of diabetes, years & 10.0 & 7.9 & 0.083 & 35 \\
Lipid panel & & & & \\
TG & 180 & 106 & 49 & 991 \\
LDL-C & 97 & 45 & 18 & 400 \\
TC & 178 & 47 & 79 & 348 \\
HDL-C & 45 & 13 & 15 & 90
\end{tabular}

10-year predicted risks for cardiovascular complication via UKPDS risk engine, $\%$

$\begin{array}{lllll}\text { CHD } & 14.6 & 11.0 & 1.4 & 57.8\end{array}$

$\begin{array}{lllll}\text { Fatal CHD } & 10.4 & 9.1 & 0.5 & 47.9\end{array}$

$\begin{array}{lllll}\text { Stroke }^{\mathrm{a}} & 6.7 & 4.8 & 0.8 & 21.6\end{array}$

\begin{tabular}{lllll} 
Fatal stroke $^{\mathrm{b}}$ & 1.0 & 0.8 & 0.1 & 3.8 \\
\hline
\end{tabular}

${ }^{a}$ Excluding outliers, $n=221$. ${ }^{b}$ Excluding outliers, $n=224$.BMI, body mass index; CHD, coronary heart disease; HDL-C, high-density lipoprotein cholesterol; LDL-C, low-density lipoprotein cholesterol; PAID, Problem Areas in Diabetes; SD, standard deviation; TC, total cholesterol; TG, triglycerides; UKPDS, United Kingdom Prospective Diabetes Study.

\section{Main results}

In the simple linear regression model, there was no statistical significance in the associations between the total PAID scores and the predicted CHD risks, fatal CHD risks, stroke risks, and fatal stroke risks; the results are summarized in Table 3. The multivariate linear regression model was constructed by incorporating covariates, and the results are summarized in Table 5. The total PAID scores were revealed to be significantly associated with the predicted 10-year fatal CHD risks ( $\mathrm{B}=0.060,95 \% \mathrm{CI}$ : [0.00084, 0.12], $\mathrm{p}=0.047$ ). For each unit increase in the total PAID score, the 10-year predicted fatal CHD risk was increased by $0.060 \%$.

\section{Discussion}

\section{Key result}

The distinction between clinical depression and diabetes distress is worth mentioning here. Clinical depression is a mental health disorder with certain symptoms (e.g., loss of motivation and feeling down); on the other hand, diabetes distress is an emotional reaction-not a mental illness-to 
Table 3: Stratified data based on baseline characteristics and UKPDS Risk Engine outputs.

\begin{tabular}{|c|c|c|c|c|c|c|c|c|c|c|}
\hline & \multicolumn{3}{|c|}{ CHD } & \multicolumn{3}{|c|}{ Fatal CHD } & \multicolumn{3}{|c|}{ Stroke } & \multirow{2}{*}{$\begin{array}{r}\text { Fatal stroke } \\
\qquad L^{\star}\end{array}$} \\
\hline & $L^{*}$ & $M^{*}$ & $\mathrm{H}^{*}$ & $L^{*}$ & $M^{*}$ & $H^{*}$ & $L^{*}$ & $M^{*}$ & $H^{*}$ & \\
\hline \multicolumn{11}{|l|}{ Gender } \\
\hline Male & 54 & 38 & 20 & 76 & 28 & 8 & 95 & 13 & 4 & 112 \\
\hline Female & 96 & 22 & 4 & 107 & 12 & 3 & 110 & 10 & 2 & 122 \\
\hline \multicolumn{11}{|l|}{ Ethnicity } \\
\hline White & 36 & 30 & 14 & 55 & 19 & 6 & 71 & 7 & 2 & 80 \\
\hline Afro-Caribbean & 81 & 9 & 1 & 86 & 4 & 1 & 81 & 7 & 3 & 91 \\
\hline Asian-Indian & 33 & 21 & 9 & 42 & 17 & 4 & 53 & 9 & 1 & 63 \\
\hline \multicolumn{11}{|l|}{ Occupational index score } \\
\hline Low (1-33) & 90 & 33 & 11 & 111 & 19 & 4 & 116 & 15 & 3 & 134 \\
\hline Medium (34-66) & 43 & 14 & 10 & 49 & 11 & 7 & 59 & 5 & 3 & 67 \\
\hline High (67-100) & 17 & 13 & 3 & 23 & 10 & 0 & 30 & 3 & 0 & 33 \\
\hline \multicolumn{11}{|l|}{ Individual annual income } \\
\hline$<\$ 20,000$ & 121 & 53 & 21 & 151 & 36 & 8 & 170 & 19 & 6 & 195 \\
\hline$\$ 20,000$ to $\$ 34,999$ & 18 & 3 & 2 & 20 & 1 & 2 & 21 & 2 & 0 & 23 \\
\hline$\$ 35,000$ to $\$ 49,999$ & 8 & 3 & 1 & 9 & 2 & 1 & 11 & 1 & 0 & 12 \\
\hline$\$ 50,000$ to $\$ 75,000$ & 2 & 1 & 0 & 2 & 1 & 0 & 2 & 1 & 0 & 3 \\
\hline$>\$ 75,000$ & 1 & 0 & 0 & 1 & 0 & 0 & 1 & 0 & 0 & 1 \\
\hline Subtotal (for each UKPDS risk engine output category) & 150 & 60 & 24 & 183 & 40 & 11 & 205 & 23 & 6 & 234 \\
\hline
\end{tabular}

*L, Low (<15\%); M, Medium (15-30\%); H, High (>30\%). CHD, coronary heart disease; UKPDS, United Kingdom Prospective Diabetes Study.

Table 4: Correlations between stratified PAID scores and UKPDS Risk Engine outputs.

\begin{tabular}{lrr}
\hline $\begin{array}{l}\text { R (correlation } \\
\text { coefficient) }\end{array}$ & $\begin{array}{r}\text { PAID score }<\mathbf{4 0} \\
(\mathbf{n}=\mathbf{1 9 5 )}\end{array}$ & $\begin{array}{r}\text { PAID score } \geq \mathbf{4 0} \\
(\mathbf{n}=\mathbf{3 9})\end{array}$ \\
\hline CHD & 0.0907 & 0.3010 \\
Fatal CHD & 0.0983 & 0.2483 \\
Stroke & 0.0250 & -0.1405 \\
Fatal stroke & 0.0294 & -0.1713 \\
\hline
\end{tabular}

CHD, coronary heart disease; PAID, Problem Areas in Diabetes; UKPDS, United Kingdom Prospective Diabetes Study.

living with diabetes. This study demonstrated that DRD was significantly associated with the predicted 10-year fatal CHD risks after controlling for covariates as noted under Table 3. These covariates ranged from baseline demographics to clinical diagnoses to medication usage factors to tobacco use. The discrepancy in statistical significance observed between simple and multivariate linear regression models can be explained by the presence of covariates that influence either or both the PAID score levels (capturing the levels of DRD) and the UKPDS Risk Engine outputs (predicting CV risks).

The finding that DRD is significantly associated with fatal CHD risks confirms similar findings from prior studies, which have identified statistically significant associations between DRD and cardiovascular heart disease [17]. Specifically, Dalsgaard and colleagues [17] determined that patients $(n=1,533)$ between the ages of 40 and 69 with type 2 diabetes mellitus, and patients with psychological distress, had a 1.7-fold higher risk of having a CVD event and a 1.8-fold higher mortality rate compared to individuals without reported distress. They also concluded that patients with higher psychological distress at the time of diagnosis had a higher risk of CVD events than individuals without reported stress [17]. Although their study did not utilize the PAID questionnaire to assess DRD levels, the overall findings still support the assumption that DRD influences the risks of adverse cardiovascular complications. Understanding this association is critical for managing the cardiovascular health of patients with diabetes. The elevated risk of cardiovascular complications at baseline for people with diabetes is well accepted among researchers and healthcare professionals. The suggested amplification of 10-year CHD risks in the presence of DRD, as shown in this study, highlights the need to classify DRD when considering possible intervention points as a potential risk factor for CVD. Table 4 shows the correlations between stratified PAID scores and the UKPDS Risk Engine outputs with varying degrees and directions; although none of the correlation coefficients are considered strong, the differences seen between high and low PAID score strata as well as between predicted CHD and stroke risks warrant further investigation.

A pilot study conducted by Young and colleagues [11] that investigated associations between DRD and predicted 
Table 5: Linear regression models.

\begin{tabular}{lrrr}
\hline & Coefficient & p-Value & 95\% Cl \\
\hline Simple linear regression models & & & \\
Predicted coronary heart disease risks vs. PAID scores & 0.016 & 0.714 & $-0.070,0.10$ \\
Predicted fatal coronary heart disease risks vs. PAID scores & 0.012 & 0.733 & $-0.059,0.083$ \\
Predicted stroke risks vs. PAID scores & -0.047 & 0.148 & $-0.111,0.017$ \\
Predicted fatal stroke risks vs. PAID scores & -0.0078 & 0.139 & $-0.018,0.0026$ \\
Multiple linear regression model* & & \\
Predicted coronary heart disease risks vs. PAID scores & 0.068 & 0.068 & $-0.0050,0.14$ \\
Predicted fatal coronary heart disease risks vs. PAID scores & 0.060 & 0.047 & $0.00084,0.12$ \\
Predicted stroke risks vs. PAID scores & 0.011 & 0.64 & $-0.037,0.060$ \\
Predicted fatal stroke risks vs. PAID scores & 0.0013 & 0.74 & $-0.0064,0.0090$ \\
\hline
\end{tabular}

${ }^{\star}$ Covariates included in this statistical model include: depression status, ethnicity, gender, age, insulin usage, number of diabetes medications, annual income, occupational index score, systolic blood pressure, BMI, and tobacco use. BMI, body mass index; CI, confidence interval; PAID, Problem Areas in Diabetes. Bold to indicate statistically significant result.

10-year cardiovascular complication risks in patients with type 2 diabetes and lower SES ( $\mathrm{n}=48$ ) also found that DRD was significantly associated with predicted fatal and nonfatal CHD risks. Specifically, this study found that with each unit increase in the total PAID score, nonfatal CHD risk was increased by $0.149 \%$, and fatal CHD risk increased by $0.129 \%$ [11]. These findings agree with the current study's results and further support the association between predicted 10-year fatal CHD risks and DRD. Also, no significant associations were found between the total PAID scores and the predicted 10-year stroke risks in the previous study [11], which agrees with this current study's results.

The impact of DRD on cardiovascular complication risks highlights the need for appropriate screening strategies and education geared toward managing psychosocial stressors. This is particularly important among those experiencing lower incomes, because they are more likely to have limited access to healthcare and diabetes management support and are at higher risk for higher baseline distress, which could impede their ability to manage their diabetes. According to Fisher and colleagues [18], 45.4\% of patients with type 2 diabetes reported moderate levels of diabetes distress, with even higher levels observed in other studies.

Additionally, identifying patients at an increased risk for DRD could benefit healthcare professionals in combating the high levels of diabetes burnout or symptoms related to distress. Delahanty et al. [19] identified that PAID scores were significantly higher among insulintreated patients than oral or diet-controlled treatments. These findings highlight the increased stress that often results from the burden of self-management and identify ways that healthcare professionals can provide education and resources for patients regarding disease expectations and individualized treatment/management goals.
Research is now being undertaken to investigate strategies that can be implemented to combat the adverse effects of DRD on diabetes management. Lee and colleagues [20] surveyed 308 veterans with type 2 diabetes on autonomy support, defined as social support for a patient's free agency to make decisions, and the effects of DRD on glycemic management. The study demonstrated a significant reduction in HbA1c over 12 months with a higher patientreported level of perceived autonomy [20]. The authors proposed that promoting autonomy for disease management from a health supporter mitigated the adverse effects of DRD on glucose management [20]. Further studies that aim to investigate implementable measures adopted by healthcare professionals, family members, and health supporters are needed for addressing the high rates of distress relating to diabetes and its potential amplification of $\mathrm{CV}$ risks in the future.

Our finding, which is generalizable to middle-aged patients with type 2 diabetes and lower SES, illustrates the significant association between DRD and predicted 10-year fatal CHD risks and provides further support for mobilizing various resources to address psychological health and medical care when treating people with diabetes. Further research dedicated to analyzing the cascade of effects that result from reducing DRD on disease-related complications and economic costs will further support the shift toward providing all-encompassing care for people with diabetes. Based on the findings of this study, healthcare professionals should be encouraged to include DRD assessment/screening as part of their routine visits and to understand the possible implications that suboptimal psychosocial well-being can have on CV and overall well-being of patients, as suggested by the 2016 ADA position statement on providing psychosocial care for those with diabetes [4]. 


\section{Relating to the practice of osteopathic medicine}

Psychosocial aspects in chronic disease evaluation and management are essential components of osteopathic physicians' practice of medicine. The osteopathic approach to patient care includes five models, e.g., biomechanical, respiratory-circulatory, metabolic-energy, neurological, and behavioral. A patient-centered approach to type 2 diabetes includes treating the whole person-e.g., integrating mental and physical health (the body-mind connection)-through preventative care, longitudinal care, timely medical interventions, and integration of structure and function. This current study integrates the assessments of participants' diabetes-related psychological states and their risks of cardiovascular events, which are essential for guiding and designing preventative and longitudinal care in a patientcentered, holistic manner for those with type 2 diabetes, combining the behavioral and metabolic-energy models of the osteopathic approach.

\section{Limitations and future directions}

The cross-sectional study design would be a limitation, because participants' data were collected only at a specific point in time without accounting for changes in their lives as time progressed. The potential step to enhance the robustness of data will be to assess participants' DRD levels utilizing the PAID questionnaire at various points with an interval of 3 months apart from each data collection; then, predictions on 10-year CV risks can be performed after every data collection. Additionally, household size data were not collected, and this information would put financial data in perspective.

Another limitation was that DRD was reflected via subjective data collected by a survey instrument. It would be possible that the statements from the PAID questionnaire were misunderstood by study participants even if the statements were read aloud to some participants who were having trouble reading and/or understanding the survey instrument.

\section{Conclusions}

In this study population with lower SES and type 2 diabetes, DRD levels and 10-year predicted fatal CHD risks exhibited a significant and positive association after adjusting for certain covariates (e.g., depression status, ethnicity, gender, age, insulin usage, number of diabetes medications, annual income, occupational index score, systolic blood pressure, BMI, and tobacco use). Physicians and other healthcare professionals are thus encouraged to incorporate a screening process for DRD in patients with type 2 diabetes in safety-net clinics as part of a routine healthcare visit. Through early detection of DRD, these patients can be supported and receive individualized approaches to psychosocial interventions that may potentially reduce their future $\mathrm{CHD}$ risks.

Acknowledgments: The authors would like to thank former PharmD students Andrew Ray and Henry Lo as well as MS student Shirley Wong during the data collection process. The research team would also like to thank Casey Shubrook, DO Candidate Class of 2025, for her contributions editing this article.

Research funding: Research funding was provided by Touro University California, Vallejo, CA.

Author contributions: All authors provided substantial contributions to conception and design, acquisition of data, or analysis and interpretation of data; all authors drafted the article or revised it critically for important intellectual content; all authors gave final approval for the version of the article to be published; and all authors agree to be accountable for all aspects of the work in ensuring that questions related to the accuracy or integrity of any part of the work are appropriately investigated and resolved.

Competing interests: None reported.

Informed consent: All participants provided written informed consent.

Ethical approval: This study was determined to be exempt from review by the Touro University California Institutional Review Board (IRB approval: P-2314).

\section{References}

1. Fisher L, Glasgow RE, Strycker LA. The relationship between diabetes distress and clinical depression with glycemic control among patients with type 2 diabetes. Diabetes Care 2010;33: 1034-6.

2. Fisher L, Skaff MM, Mullan JT, Arean P, Mohr D, Masharani U. Clinical depression versus distress among patients with type 2 diabetes: not just a question of semantics. Diabetes Care 2007; 30:542-8.

3. Kreider KE. Diabetes distress or major depressive disorder? A practical approach to diagnosing and treating psychological comorbidities of diabetes. Diabetes Ther 2017;8:1-7.

4. Young-Hyman D, de Groot M, Hill-Briggs F, Gonzales JS, Hood K, Peyrot M. Psychosocial care for people with diabetes: a position statement of the American Diabetes Association. Diabetes Care 2016;39:2126-40. 
5. Ohrnberger J, Fichera E, Sutton M. The relationship between physical and mental health: a mediation analysis. Soc Sci Med 2017;195:42-9.

6. Peyrot M, Rubin RR, Lauritzen T, Snoek FJ, Matthews DR, Skovlund SE. Psychosocial problems and barriers to improved diabetes management: results of the cross-national Diabetes Attitudes, Wishes and Needs (DAWN) Study. Diabet Med 2005;22:1379-85.

7. Rotella F, Mannucci E. Diabetes mellitus as a risk factor for depression. A meta-analysis of longitudinal studies. Diabetes Res Clin Pract 2013;99:98-104.

8. Nicolucci A, Burns KK, Holt RIG, Comachi M, Hermanns N, Ishii H. Diabetes Attitudes, Wishes and Needs second study (DAWN $2^{\mathrm{TM}}$ ): cross-national benchmarking of diabetes-related psychosocial outcomes for people with diabetes. Diabet Med 2013;30: 767-77.

9. Winchester RJ, Williams JS, Wolfman TE, Egede LE. Depressive symptoms, serious psychological distress, diabetes distress and cardiovascular risk factor control in patients with type 2 diabetes. J Diabetes Complications 2016;30:312-7.

10. Farooqi A, Khunti K, Abner S, Gillies C, Morriss R, Seidu S. Comorbid depression and risk of cardiac events and cardiac mortality in people with diabetes: a systematic review and metaanalysis. Diabetes Res Clin Pract 2019;156:107816.

11. Young CF, Cheng J, McCarter G. Associations between diabetesrelated distress and cardiovascular complication risks in patients with type 2 diabetes and lower socioeconomic status: a pilot study. Diabetes Spectr 2019;32:257-63.

12. Pernomian L, Moreira JD, Gomes MS. In the view of endothelial microparticles: novel perspectives for diagnostic and pharmacological management of cardiovascular risk during diabetes distress. J Diabetes Res 2018;2018:9685205.
13. Polonsky WH, Anderson BJ, Lohrer PA, Welch G, Jacobson AM, Aponte JE. Assessment of diabetes-related distress. Diabetes Care 1995;18:754-60.

14. UK Prospective Diabetes Study (UKPDS) Risk Engine. University of Oxford, Diabetes Trials Unit. Available from: https://www.dtu.ox. ac.uk/riskengine/ [Accessed 30 Nov 2020].

15. Nam CB, Boyd M. Occupational status in 2000: over a century of census-based measurement. Popul Res Policy Rev 2004;23: 327-58.

16. Baron S. Measuring occupation as an element of socioeconomic status/position (Presentation). Department of Health and Human Services, National Institute for Occupational Safety and Health, and Centers for Disease Control and Prevention. Available from: http://www.ncvhs.hhs.gov/120308p2.pdf [Accessed 30 Nov 2020].

17. Dalsgaard EM, Vestergaard M, Skriver MV, Maindal HT, Lauritzen T, Borch-Johnsen K. Psychological distress, cardiovascular complications and mortality among people with screen-detected type 2 diabetes: follow-up of the ADDITION-Denmark trial. Diabetologia 2014;57:710-7.

18. Fisher L, Hessler DM, Polonsky WH, Mullan J. When is diabetes distress clinically meaningful? Establishing cut points for the Diabetes Distress Scale. Diabetes Care 2012;35:259-64.

19. Delahanty LM, Grant RW, Wittenberg E, Bosch JL, Wexler DJ, Cagliero E. Association of diabetes-related emotional distress with diabetes treatment in primary care patients with Type 2 diabetes. Diabet Med 2007;24:48-54.

20. Lee AA, Piette JD, Heisler M, Rosland A. Diabetes distress and glycemic control: the buffering effect of autonomy support from important family members and friends. Diabetes Care 2018;41: 1157-63. 